\title{
Detection of QTL for phosphorus efficiency and biomass traits at the seedling stage in wheat
}

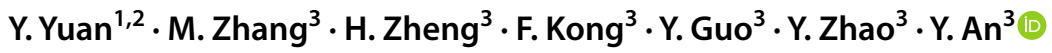

Received: 21 April 2020 / Accepted: 24 July 2020 / Published online: 27 August 2020

(c) The Author(s) 2020

\begin{abstract}
Phosphorus $(\mathrm{P})$ is one of the most vital nutrient elements in crop output and quality formation. In this study, four biomass, four P uptake efficiency (PupE), and three P-utilization efficiency (PutE) traits were investigated using a set of recombinant inbred lines (RILs) derived from a cross of "SN0431 $\times$ LM21", under hydroponic culture trials at low P (LP) and normal P (NP) levels in two different seasons, respectively. A total of 85 QTL were identified on 18 chromosomes except for 1D, 2A, and 3D. Among them, 36 and 42 QTL were detected under LP and NP, respectively, and seven QTL were simultaneously detected under LP and NP. Seventeen relatively high-frequency QTL (RHF-QTL) were detected. The average contributions of 13 major RHF-QTL were over 10.00\%. Five important QTL clusters were detected on chromosomes 4D, 5A, and 5B. Among them, positive linkages were observed between PutE and biomass traits at four QTL clusters, C1, C2, C3, and C6, showing these loci may be hot spots for genetic control of both phosphorus utilization and biomass accumulation in wheat seedlings. In addition, correlation analysis indicated that three biomass traits (SDW, RDW, and TDW) should be used as primary selection indexes for PE at the seedling stage.
\end{abstract}

Keywords Wheat · Phosphorus efficiency (PE) · Morphological trait · Quantitative trait loci (QTL) · Recombinant inbred line (RIL)

\begin{tabular}{ll}
\multicolumn{2}{l}{ Abbreviations } \\
QTL & Quantitative trait loci \\
RIL & Recombinant inbred line \\
RHF-QTL & Relatively high-frequency QTL \\
PE & Phosphorus efficiency \\
PupE & Phosphorus uptake efficiency \\
PutE & Phosphorus utilization efficiency \\
SDW & Shoot dry weight per plant \\
RDW & Root dry weight per plant
\end{tabular}

Communicated by V. Korzun.

Electronic supplementary material The online version of this article (https://doi.org/10.1007/s42976-020-00067-4) contains supplementary material, which is available to authorized users.

Y. An

anyr@sdau.edu.cn

1 Yantai Academy of Agricultural Sciences, Yantai 265500, Shandong, China

2 Jinan Academy of Agricultural Sciences, Jinan 205316, Shandong, China

3 State Key Laboratory of Crop Biology, Shandong Agricultural University, Tai' an 271018, Shandong, China

$\begin{array}{ll}\text { TDW } & \text { Total dry weight per plant } \\ \text { RSDW } & \text { Ratio of root and shoot dry weight } \\ \text { SPC } & \text { Shoot P-content per plant } \\ \text { RPC } & \text { Root P-content per plant } \\ \text { TPC } & \text { Total P-content per plant } \\ \text { RSPC } & \text { Ratio of root and shoot P-content } \\ \text { SPutE } & \text { Shoot P-utilization efficiency } \\ \text { RPutE } & \text { Root P-utilization efficiency } \\ \text { TPutE } & \text { Total P-utilization efficiency }\end{array}$

\section{Introduction}

Phosphorus $(\mathrm{P})$ is one of the most vital nutrient elements in crop output and quality formation, not only presenting in major organic molecules (e.g., RNA, DNA, ATP, and membrane phospholipids) but also playing important roles in photosynthesis, energy metabolism, enzymatic reactions, and sugar metabolism (Péret et al. 2011; Qiu et al. 2013). P is the second most limiting nutrient for crop production after $\mathrm{N}$ (Bernardino et al. 2019). A large amount of $P$ fertilizer has been used to achieve higher crop yields and ensure food security worldwide (Dhillon et al. 2017). P fertilizer application has tripled over 
the past three decades in the North China Plain, where it produces almost $60-80 \%$ of China's wheat (Triticum aestivum $\mathrm{L}$.) (Xin et al. 2017). In addition, the $P$ fertilizer use efficiency of wheat is only approximately $10.7 \%$, which is lower than that of rice (13.1\%) and maize (11.0\%) (Ma et al. 2011). High $P$ inputs and low use efficiency have led to $P$ resource exhaustion and environmental pollution (Bayuelo-Jiménez et al. 2011; Dawson and Hilton 2011). Hence, it is necessary and significant to excavate the genetic potential of phosphorus efficiency $(\mathrm{PE})$ in wheat for saving phosphorus resources and protecting ecological environment (Baker et al. 2015; Vandamme et al. 2016).

$\mathrm{PE}$ relates to the ability of wheat to recover $\mathrm{P}$ from fertilizer and soil, which can be attributed to two factors: P uptake efficiency (PupE) and P-utilization efficiency (PutE) (Meng et al. 2014; Siddiqi and Glass 1981). The volume of publications on the genotypic variation of PE has been widely documented in wheat (Liao et al. 2008; Malhi et al. 2015; Nisar et al. 2016), which provide a rich material basis for studying the genetic basis of PE. Furthermore, PE and associated traits are complex quantitative traits controlled by multiple genes (Wang et al. 2018). Quantitative trait loci (QTL) mapping is an effective tool commonly used in studies on the inheritance of quantitative traits. With recent advances in molecular marker technology and high-throughput genotyping systems (Gunderson et al. 2005; Syvänen 2005), a number of QTL for PE and related traits have been published in recent decades (Su et al. 2006; Ryan et al. 2015), including some relatively high-frequency QTL (RHF-QTL) and QTL clusters in the same chromosome regions (Guo et al. 2012; Zhang and Wang 2015). However, only a few papers have concerned and detected important QTL clusters containing one or more RHF-QTL and QTL loci that control both PE and biomass traits (Su et al. 2009; Yuan et al. 2017). Important QTL clusters including stable QTL in several environments and knowledge regarding the relationship among PE and biomass traits at QTL level will be crucial to further understand the effects of $\mathrm{P}$ on growth in wheat.

In this study, we used a set of RILs to investigate four biomass, three PupE, and three PutE traits under low and normal $\mathrm{P}$ concentrations in hydroponic culture across two continuous growing seasons. The objectives were to detect QTL with significant contributions to PE and biomass traits, to find RHF-QTL and important QTL clusters, and to identify QTL locus controlling both PE and biomass traits at the seedling stage.

\section{Materials and methods}

\section{Plant materials}

A total of 176 lines were selected from a set of RILs derived from a cross "Shannong $0431($ SN0431) $\times$ Lumai
21 (LM21)" (SL-RILs, $F_{10}$ in 2013) by single-seed descent (SSD). SN0431 is a germplasm developed by our group using an $F_{3}$ cross of "Grtpi85504//MI76-77-S29/ALD" from the International Winter $\times$ Spring Wheat Screening Nursery (IWSWSN), 1994, Cornell University, USA. LM21 is derived from a cross of "Yanzhong 144/Baofeng 7228" and released by the Yantai Academy of Agricultural Science of China in 1996. LM21 has been planted on more than 30 million hectares in the Huang-huai Winter Wheat Region and the Northern Winter Wheat Region of China. The two parents have distinct differences in PE and biomass traits at seedling stage. Specifically, SN0431 is obviously better than LM21 in four biomass traits, including RDW, SDW, TDW, and RSDW, four PupE traits, involving SPC, RPC, TPC and RSPC, and three PutE traits, containing SPutE, RPutE and TPutE under low and normal $P$ conditions in two different trials (Table S1).

\section{Experimental design}

Two hydroponic culture trials were conducted from November 25 to December 31 in 2013 (Trial 1) and from March 2 to April 6 in 2014 (Trial 2) in the greenhouse at Shandong Agricultural University. The 176 RILs and their parents were grown under LP and NP concentrations in each trial. Thus, four treatments, LP1, NP1, LP2, and NP2, were designed (Table 1). Four replications were set up for each treatment, with two uniform seedlings for each RI line. A random complete block design was used in the experiments with four replications for each treatment. Modified nutrient solution (Guo et al. 2012) was used to optimize wheat growth (Table S2) using Hoagland nutrient solution (Hoagland and Arnon 1950). The detailed culture methods were as described in our previous study (Kong et al. 2013).

To obtain more robust seedlings, a 9-h photoperiod was employed. The temperature, relative humidity and light intensity information was collected and recorded every 10 min by the ZDR Data Loggers (Zhejiang University Electric Equipment Factory, China). In Trial 1, the temperature, relative humidity and light intensity varied from 8.1 to

Table $1 \mathrm{P}$ treatments for the hydroponic culture trials

\begin{tabular}{llllll}
\hline Years & Trials & Names & Treatments & Times & $\begin{array}{l}P \text { concen- } \\
\text { trations } \\
(\mathrm{mM})\end{array}$ \\
\hline 2013 & 1 & $\mathrm{NP}$ & $\mathrm{NP} 1$ & $1 \times \mathrm{P}$ & 0.2 \\
& & LP & LP1 & $1 / 10 \times \mathrm{P}$ & 0.02 \\
2014 & 2 & NP & NP2 & $1 \times \mathrm{P}$ & 0.2 \\
& & LP & LP2 & $1 / 10 \times \mathrm{P}$ & 0.02 \\
\hline
\end{tabular}

$L P$ low phosphorus, $N P$ normal phosphorus, $N P 1$ and $N P 2$ normal phosphorus treatment in trial 1 and trial 2 respectively, $L P 1$ and $L P 2$ low phosphorus treatment in trial 1 and trial 2 respectively 
$30.7{ }^{\circ} \mathrm{C}, 11.9$ to $69.7 \%$ and 0.0 to 47.8 klux, respectively, and the average values were $16.0{ }^{\circ} \mathrm{C}, 49.4 \%$, and $4.1 \mathrm{klux}$, respectively. In Trial 2 , the temperature, relative humidity and light intensity changed from $8.0{ }^{\circ} \mathrm{C}$ to $36.8^{\circ} \mathrm{C}, 5.0$ to $82.9 \%$ and 0.0 to 50.4 klux, respectively, and the mean values were $19.8{ }^{\circ} \mathrm{C}, 33.4 \%$, and $6.3 \mathrm{klux}$, respectively. Samples have been taken when the seedlings have grown to four leaves and one heart, corresponding to 14 and 20 for $\mathrm{BBCH}$. Because of the huge quantity of work for the measurement of $\mathrm{P}$ concentrations, all the individual plants of each line for the four replications in the same $\mathrm{P}$ concentration treatment were harvested together as one mixed sample and separated into two parts: root and shoot. Then, all the samples were oven-dried at $60{ }^{\circ} \mathrm{C}$ for $72 \mathrm{~h}$ to constant mass.

\section{Trait measurement}

Four biomass traits (SDW, RDW, TDW, and RSDW), four PupE traits (SPC, RPC, TPC, and RSPC) and three PutE traits (SPutE, RPutE, and TPutE) were investigated. The mixed samples of the same $P$ treatment were used to measure the shoot dry weight per plant (SDW) and root dry weight per plant (RDW). After that, we milled all the plant samples. Dried tissue samples were digested using concentrated $\mathrm{H}_{2} \mathrm{SO}_{4}$ and $\mathrm{H}_{2} \mathrm{O}_{2}$ until the mixture was clear. The P-contents for the shoots or roots of each plant (SPC and RPC) were measured using a UV/VIS spectrometer (PerkinElmer Lambda 25, USA), and the colorimetric wavelength was $450 \mathrm{~nm}$. A summary of the trait measurement and calculation (Guo et al. 2012; Siddiqi and Glass 1981) for all 11 traits is listed in Table 2.

\section{Data analysis}

SPSS 18.0 software (SPSS Inc., Chicago, IL, USA) was employed to conduct analyses of variance (ANOVA), the least significant difference (LSD) test, and Spearman's correlation coefficients $(r)$ between different traits. In a no-repeat trial design, a two-factor model was adequate for ANOVA. All factors, including RILs $(n-1)$ degrees of freedom, treatments $(t-1)$, and random error $[(n-1)(t-1)]$, were considered sources of random effects. Multiple comparison tests for the traits between "treatments" were calculated by considering all the RILs as replicates and using the mean value of the same $\mathrm{P}$ condition for each trait. The variance of $\mathrm{P}$ conditions was excluded when the broad-sense heritability $\left(h_{B}^{2}\right)$ was estimated according to the formula $h_{B}^{2}=\sigma_{g}^{2} /\left(\sigma_{g}^{2}+\sigma_{e}^{2}\right)$, where $\sigma_{g}^{2}$ was the genotypic variance and $\sigma_{e}^{2}$ was the total error variance.

A high-density genetic map based on SL-RILs (Gao 2014, Table S3, Fig. S1) was employed in the QTL analysis. The map was constructed with 5916 loci covering all 21 chromosomes. Of these loci, 4530 were unique, and the other 1386 showed cosegregation with other markers. The final map spanned a total length of $2929.96 \mathrm{cM}$ across 42 linkage groups, with a marker density of $0.65 \mathrm{cM}$. Windows QTL Cartographer 2.5 software (http://statgen.ncsu.edu/ qtlcart/WQTLCart.htm) was used to perform the QTL mapping, and composite-interval mapping (CIM) was selected to search for QTL of each trait separately for each of two different environments (LP1, NP1, LP2, and NP2) and for the average value of the same $P$ level in Trail 1 and Trial 2 (LPAV and NPAV). The CIM analysis was completed using the standard model (Model 6) with a walk speed of $0.5 \mathrm{cM}$. "Forward and backward regression" was used to control the genetic background. The control marker number and the blocked window size were 5 and $10 \mathrm{cM}$. The empirical LOD thresholds were estimated using 1000 permutations at $p \leq 0.05$ (Churchill and Doerge 1994), and the LOD threshold value of different trait-treatment combinations varied from 3.20 to 4.03. Considering the effects of phosphorus stress, we defined a RHF-QTL or stable QTL when it was

Table 2 Summary of investigated traits and their measurement methods under hydroponic culture trials

\begin{tabular}{|c|c|c|c|}
\hline \multicolumn{2}{|l|}{ Traits } & \multirow{2}{*}{$\begin{array}{l}\text { Units } \\
\text { mg plant }^{-1}\end{array}$} & \multirow{2}{*}{$\begin{array}{l}\text { Methods of measurement } \\
\text { Oven-dried and weighted using } 1 / 10000 \text { balances }\end{array}$} \\
\hline SDW & Shoot dry weight per plant & & \\
\hline RDW & Root dry weight per plant & mg plant ${ }^{-1}$ & Oven-dried and weighted using 1/10000 balances \\
\hline TDW & Total dry weight per plant & mg plant ${ }^{-1}$ & $\mathrm{RDW}+\mathrm{SDW}$ \\
\hline RSDW & Ratio of root and shoot dry weight & - & RDW/SDW \\
\hline SPC & Shoot P-content per plant & mg plant ${ }^{-1}$ & Using a UV/VIS spectrometer (PerkinElmer Lambda \\
\hline $\mathrm{RPC}$ & Root P-content per plant & $\mathrm{mg} \mathrm{plant}^{-1}$ & $\begin{array}{l}\text { 25, USA).and the colorimetric wavelength was } \\
450 \mathrm{~nm}\end{array}$ \\
\hline TPC & Total P-content per plant & mg plant ${ }^{-1}$ & $\mathrm{RPC}+\mathrm{SPC}$ \\
\hline RSPC & Ratio of root and shoot P-content & - & $\mathrm{RPC} / \mathrm{SPC}$ \\
\hline SPutE & Shoot P-utilization efficiency & $\mathrm{mg}\left(\mu \mathrm{g} \mathrm{mg}^{-1}\right)^{-1}$ & $\mathrm{SDW} /[(\mathrm{SPC} \times 1000) / \mathrm{SDW}]$ \\
\hline RPutE & Root P-utilization efficiency & $\mathrm{mg}\left(\mu \mathrm{g} \mathrm{mg}^{-1}\right)^{-1}$ & $\mathrm{RDW} /[(\mathrm{RPC} \times 1000) / \mathrm{RDW}]$ \\
\hline TPutE & Total P-utilization efficiency & $\mathrm{mg}\left(\mu \mathrm{g} \mathrm{mg}^{-1}\right)^{-1}$ & $\mathrm{TDW} /[(\mathrm{TPC} \times 1000) / \mathrm{TDW}]$ \\
\hline
\end{tabular}


detected in two or more treatments ( $1 / 3$ of all six treatments) for the hydroponic culture trials. We defined a QTL cluster as three or more traits with significant QTL having overlapping confidence intervals (Gong et al. 2015). We confirmed the confidence intervals for an RHF-QTL or a QTL cluster by meta-QTL analysis using Biomercator 2.0 software and used $\mathrm{AIC}=4$ (model 4$)$ in the step Meta-analysis $2 / 2$ (http://www.genoplante.com).

\section{Results}

\section{Phenotypic variation and the effects of low $P$ input on seedling traits}

The female parent of the RILs, SN 0431, was obviously superior to the male parent LM21 in all 11 investigated traits in different treatments (Table S1). For the RIL population, transgressive segregation was observed for all 44 trait-treatment combinations. The coefficients of variation $(\mathrm{CVs} ; \mathrm{CV}=\mathrm{SD} /$ Average $\times 100 \%)$ ranged from 11.93 (RSDW in LP1) to $71.29 \%$ (TPutE in LP1). All 11 traits in each trait-treatment combination exhibited a continuous distribution.

The results of ANOVA showed that the variance for either genotype or treatment effects on all tested traits was significant at $p \leq 0.001$ (Table S4), indicating that the genetic background and different $\mathrm{P}$ treatments were both important in explaining the overall phenotypic variations. The $h_{B}^{2}$ for the targeted traits changed from 58.21 (RSDW in Trial 2) to 90.43\% (TDW in Trial 1) (Table S4).

According to the results of the LSD test, the average values of the 11 seedling traits were in most cases significantly different between the LP and NP treatments, except for RDW in Trial 2 (Table S1). Specifically, there was a significant trend with the reduction of $\mathrm{P}$ concentration in the nutrient solution going with lower SDW, TDW, SPC, RPC, and TPC. Nevertheless, as the P concentration decreased, RSDW, RSPC, RPutE, SPutE, and TPutE increased significantly. These results demonstrated that low $\mathrm{P}$ input could intensely inhibit the accumulation of biomass and $\mathrm{P}$ element but enhance the ratio of root and shoot dry weight and the P-utilization efficiency at the seedling stage.

\section{Correlations between tested traits}

Using the average values of four $\mathrm{P}$ treatments for each trait, the Spearman correlation coefficients $(r)$ among the 11 targeted traits were almost all significant $(p \leq 0.05)$ or extremely significant $(p \leq 0.01)$, except for the $r$ between RSPC and SDW/TDW, SPutE and RSDW/SPC/TPC, TPutE and TPC (Table S5). An extremely significant positive correlation was found between three biomass traits (SDW,
RDW, and TDW) and six PE traits (SPC, RPC, TPC, SPutE, RPutE, and TPutE), suggesting that seedling biomass traits can reflect the capacity of phosphorus absorption and utilization in wheat to some extent.

\section{Located QTL}

A total of 85 additive QTL for the 11 traits investigated (108 QTL for trait-treatment combinations) were detected on 18 chromosomes except for 1D, 2A, and 3D (Table S6, Fig. S1). A number of 36 and 42 QTL were detected only under LP and NP conditions, respectively, and seven QTL were simultaneously found under LP and NP conditions. For the biomass traits (RDW, SDW, RSDW and TDW), PupE traits (RPC, SPC, RSPC, and TPC), and PutE traits (RPutE, SPutE, and TPutE), 34, 30, and 21 QTL were identified, respectively. An individual QTL explained 6.41\% ( $Q S p c-7 A$ in NP1 treatment) to $19.55 \%$ ( $Q R s p c-6 D$ in NPAV treatment) of the phenotypic variation, and the highest LOD value for single QTL was 10.36 for QSpute-5B.1 in the NPAV treatment. There were 28 QTL that showed positive additive effects with SN0431 increasing the effects of QTL, whereas 57 QTL had negative additive effects with LM21 increasing the QTL effects.

\section{RHF-QTL and QTL clusters}

A total of 17 RHF-QTL were detected for 9 traits (except for RDW and RPutE) on chromosomes 1B, 4D, 5A, 5B, 5D, 6A, and 6B (Table 3, Fig.S1), accounting for 37.04\% $(40 / 108 \times 100 \%)$ of the QTL for trait-treatment combinations. The average contributions ranged from 8.41 to $15.50 \%$. Of these, the average contributions of 13 RHFQTL were over $10.00 \%$, indicating that they were major RHF-QTL. The additive effects of two QTL (QSpc-1B.1 and $Q R s d w-5 D$ ) were positive with increasing QTL effects from SN0431, whereas the other 15 RHF-QTL were negative with increasing QTL effects from LM21. Seven RHFQTL, QSdw-5B.1, QSdw-5B.4, QTdw-5B.1, QTdw-5B.4, QSpute-4D, QSpute-5A, and QTpute-4D, were expressed in both the LP and NP treatments simultaneously, which implied that these QTL were less affected by different phosphorus conditions.

A number of the seven QTL clusters (C1-C7) were mapped on chromosomes 4D, 5A, and 5B (Table 4, Fig. $\mathrm{S} 1$ ). These clusters were associated with ten out of 11 traits investigated (without RSPC) and involved $44.44 \%$ of the QTL $(48 / 108 \times 100 \%)$ for trait-environment combinations. In particular, 12 RHF-QTL were detected in five QTL clusters $(\mathrm{C} 1, \mathrm{C} 2, \mathrm{C} 3, \mathrm{C} 6$, and $\mathrm{C} 7)$, which were considered to be important QTL clusters. 
Table 3 Relatively high-frequency QTL (RHF-QTL) detected in two or more treatments under hydroponic culture trials

\begin{tabular}{|c|c|c|c|c|c|c|c|c|c|}
\hline \multirow[t]{2}{*}{ Traits } & \multirow[t]{2}{*}{ QTL } & \multirow[t]{2}{*}{ Treatments } & \multirow[t]{2}{*}{ Marker interval } & \multicolumn{3}{|c|}{ Additive effects } & \multicolumn{3}{|l|}{$R^{2}(\%)$} \\
\hline & & & & Max & Min & Average & Max & Min & Average \\
\hline \multirow[t]{3}{*}{ SDW } & $Q S d w-4 D$ & LP1, LP2, LPAV & $D-1124727-D-1108531$ & -3.16 & -4.4 & -3.78 & 11.13 & 8.39 & 9.76 \\
\hline & $Q S d w-5 B .1$ & LPAV, NPAV & $S-2283173-D-3031624$ & -3.65 & -4.6 & -4.13 & 12.6 & 8.52 & 10.56 \\
\hline & $Q S d w-5 B .4$ & LPAV, NPAV & $D-100091085-D-100010859$ & -3.16 & -3.7 & -3.43 & 8.41 & 8.41 & 8.41 \\
\hline RSDW & $Q R s d w-5 D$ & NP2, NPAV & $D-1218894-D-1243224$ & 0.01 & 0.01 & 0.01 & 11.43 & 11.01 & 11.22 \\
\hline \multirow[t]{3}{*}{ TDW } & $Q T d w-5 A$ & NP2, NPAV & $D-1282770-D-1117080$ & -5.7 & -6.54 & -6.12 & 14.71 & 11.08 & 12.9 \\
\hline & $Q T d w-5 B .1$ & NP2, LPAV, NPAV & $D-3023523-D-3020625$ & -4.73 & -6.86 & -5.62 & 17.7 & 6.76 & 11.73 \\
\hline & $Q T d w-5 B .4$ & LPAV, NPAV & $D-100091085-D-3033994$ & -3.98 & -4.34 & -4.16 & 9.47 & 8.65 & 9.06 \\
\hline RPC & $Q R p c-6 A$ & NP2, NPAV & $S-2310594-D-1859763$ & -0.01 & -0.01 & -0.01 & 10.01 & 7.87 & 8.94 \\
\hline SPC & $Q S p c-1 B .1$ & NP2, NPAV & $D-1258454-D-1110478$ & 0.07 & 0.04 & 0.06 & 11.33 & 8.94 & 10.14 \\
\hline RSPC & $Q R s p c-6 D$ & NP2, NPAV & $D-100003132-D-1676641$ & -0.01 & -0.01 & -0.01 & 19.55 & 11.45 & 15.5 \\
\hline TPC & $Q T p c-5 B$ & NP2, NPAV & $D-1065599-D-1107756$ & -0.05 & -0.07 & -0.06 & 11.65 & 10.21 & 10.93 \\
\hline \multirow[t]{3}{*}{ SPutE } & QSpute-4D & LP1, NP2, LPAV, NPAV & $D-1124727-D-1108531$ & -0.55 & -0.71 & -0.63 & 12.79 & 8.38 & 10.59 \\
\hline & QSpute-5A & NP2, NP1, LPAV, NPAV & $D-1282770-S-1127707$ & -0.56 & -0.72 & -0.63 & 15.49 & 8.5 & 11.77 \\
\hline & QSpute-5B.1 & NP2, NPAV & $S-2283173-D-100082275$ & -0.58 & -0.75 & -0.67 & 17.93 & 9.95 & 13.94 \\
\hline \multirow[t]{3}{*}{ TPutE } & QTpute- $4 D$ & LP1, NP2 & $D-1124727-D-1108531$ & -0.64 & -0.9 & -0.77 & 15.1 & 8.58 & 11.84 \\
\hline & QTpute-5A.2 & NP1, NPAV & $D-100041125-S-1127707$ & -0.58 & -0.66 & -0.62 & 12.86 & 8.4 & 10.63 \\
\hline & QTpute-5B.1 & NP2, NPAV & $S-2283173-D-100082275$ & -0.65 & -0.84 & -0.75 & 16.99 & 9.56 & 13.28 \\
\hline
\end{tabular}

$N P 1$ and $N P 2$ normal phosphorus treatment in trial 1 and trial 2 respectively, $L P 1$ and $L P 2$ low phosphorus treatment in trial 1 and trial 2 respectively, NPAV the average value of normal phosphorus level in trial 1 and trial $2, L P A V$ the average value of low phosphorus level in trail 1 and trail 2

\section{Discussion}

\section{Effects of LP and preliminary evaluation index of PE at wheat seedling stage}

In the present study, the LP treatment significantly reduced SDW, TDW, SPC, RPC, and TPC but prominently increased RSDW, RSPC, RPutE, SPutE, and TPutE compared with the NP treatments. This result demonstrated that the LP treatment can inhibit the growth of biomass and the PupE, whereas it can promote more nutrient transport in roots than in shoots and result in higher $\mathrm{P}$ efficient utilization in wheat seedlings. It is in accordance with that nutrient cycling between roots and shoots is significantly regulated by nutrient supply levels (López-Bucio et al. 2003; Schachtman et al. 1998).

In the present study, we found that the $r$ values between three biomass traits (SDW, RDW, and TDW) and six PE traits (SPC, RPC, TPC, SPutE, RPutE, and TPutE) were all significantly positive, indicating that the higher the biomass, the higher the PupE and PutE at the seedling stage. Thus, the three biomass traits (SDW, RDW, and TDW) could be used as the primary morphological indexes for the evaluation of PE at seedling stage avoiding testing P-content of all germplasm, so as to improve the screening efficiency of wheat phosphorus efficient germplasm.

\section{QTL locations for PE and related traits}

In recent decades, some studies of QTL location for wheat morphological and PE-related traits have been detected and mapped on all 21 chromosomes. Most of the QTL have been identified under conditions of $\mathrm{P}$ deficiency and adequacy in hydroponic culture trial (Guo et al. 2012; Zhang and Wang 2015), pot trials (Ryan et al. 2015; Su et al. 2006), and field trials (Su et al. 2009).

In the present study, we found 85 additive QTL for 11 biomass- and PE-associated traits under low and normal $\mathrm{P}$ conditions. Some similar QTL have been mapped in the same or adjacent marker regions compared with previous QTL mapping results. We located a QTL for RPC in the marker interval wPt-672089-wPt-667155 on chromosome $1 \mathrm{~A}$, and a QTL for thousand-kernel weight using WY populations was co-located in the region (Cui et al. 2014). In this study, a QTL for RSPC was detected in the marker interval wPt-6156-S-1093076 on chromosome 7B, and a QTL for grain number per spike (Yuan et al. 2017) and a QTL for shoot potassium concentration (Guo et al. 2012) were co-located in this region. However, because of the distinct component markers and different genetic backgrounds, most of the QTL in this study were linked with new markers.

Different $P$ input levels can greatly affect the expression of the QTL for PE and biomass traits (Zhang and Wang 2015). Of all the 85 QTL under different $P$ concentrations, 
Table 4 QTL clusters for more than three traits at seedling stages

\begin{tabular}{|c|c|c|c|c|c|c|c|}
\hline Codes & $\begin{array}{l}\text { Chromo- } \\
\text { somes }\end{array}$ & Marker intervals & No. of QTL & QTL & Treatments & Additive effects & $R^{2}(\%)$ \\
\hline \multirow[t]{5}{*}{$\mathrm{C} 1$} & $4 \mathrm{D}$ & $D-1124727-D-1108531$ & 5 & $Q S d w$ & LP1, LP2, LPAV & -3.78 & 9.76 \\
\hline & & & & $Q T d w$ & LPAV & -4.11 & 9.91 \\
\hline & & & & $Q R s d w$ & LPAV & 0.01 & 9.74 \\
\hline & & & & QSpute & LP1,NP2,NPAV, LPAV & -0.63 & 10.59 \\
\hline & & & & QTpute & LP1,NP2 & -0.77 & 11.84 \\
\hline \multirow[t]{5}{*}{$\mathrm{C} 2$} & $5 \mathrm{~A}$ & $D-1282770-S-985909$ & 5 & $Q S d w$ & NPAV & -4.83 & 13.80 \\
\hline & & & & $Q T d w$ & NP2, NPAV & -6.54 & 11.08 \\
\hline & & & & QSpute & NP1, NP2, LPAV, NPAV & -0.63 & 11.77 \\
\hline & & & & QTpute.1 & NP2 & -0.84 & 15.62 \\
\hline & & & & QRpute.2 & NPAV & -0.12 & 13.65 \\
\hline \multirow[t]{5}{*}{$\mathrm{C} 3$} & $5 \mathrm{~B}-1$ & $S-2283173-D-100082275$ & 5 & $Q S d w .1$ & NPAV, LPAV & -4.60 & 12.60 \\
\hline & & & & $Q T d w .1$ & NP2, NPAV, LPAV & -5.27 & 6.76 \\
\hline & & & & QRpute.2 & NPAV & -0.17 & 11.22 \\
\hline & & & & QSpute.1 & NP2, NPAV & -0.58 & 9.95 \\
\hline & & & & QTpute.1 & NP2, NPAV & -0.65 & 9.56 \\
\hline \multirow[t]{4}{*}{$\mathrm{C} 4$} & $5 \mathrm{~B}-1$ & $S-2259846-D-2322388$ & 4 & $Q S d w .2$ & NP1 & -5.96 & 15.63 \\
\hline & & & & $Q T d w .2$ & NP1 & -6.99 & 16.08 \\
\hline & & & & QSpute.2 & NP1 & -0.92 & 16.18 \\
\hline & & & & QTpute.2 & NP1 & -0.99 & 14.49 \\
\hline \multirow[t]{3}{*}{ C5 } & $5 \mathrm{~B}-2$ & $D-1078595-D-1249528$ & 3 & $Q T d w .3$ & LP2 & -5.44 & 10.26 \\
\hline & & & & QSpute.3 & LP2 & -1.01 & 10.32 \\
\hline & & & & QTpute.3 & LP2 & -1.31 & 12.26 \\
\hline \multirow[t]{5}{*}{ C6 } & $5 \mathrm{~B}-2$ & $D-100091085-D-3033994$ & 5 & $Q R d w .2$ & LPAV & -0.56 & 7.39 \\
\hline & & & & $Q S d w .4$ & NPAV, LPAV & -5.96 & 15.63 \\
\hline & & & & $Q T d w .4$ & NPAV, LPAV & -4.34 & 8.65 \\
\hline & & & & QSpute.4 & NPAV & -0.42 & 6.73 \\
\hline & & & & QTpute.4 & NPAV & -0.48 & 6.57 \\
\hline \multirow[t]{3}{*}{ C7 } & $5 \mathrm{~B}-2$ & $w P t-666268-S-1036667$ & 3 & $Q R p c .2$ & NP2 & -0.01 & 8.10 \\
\hline & & & & $Q S p c .4$ & NPAV & -0.04 & 11.26 \\
\hline & & & & $Q T p c$ & NP2, NPAV & -0.07 & 10.21 \\
\hline
\end{tabular}

$36(42.35 \%)$ and 42 (49.41\%) QTL were detected specifically under LP and NP treatment(s), respectively. Among them, one RHF-QTL, $Q S d w-4 D$, was expressed under all LP treatments (LP1, LP2, and LPAV), and nine RHF-QTL were identified under two NP treatments (Table 3 and Table S6). Only seven RHF-QTL ( $Q S d w-5 B .1, Q S d w-5 B .4, Q T d w-$ 5B.1, QTdw-5B.4, QSpute-4D, QSpute-5A, and QTpute-4D) could function under both LP and NP conditions (Table 3 and Table S6), and their appearance was less affected by different $P$ supply levels. Similar results have been found in previous studies as well (Gong et al. 2015; Guo et al. 2012; Kong et al. 2013).

\section{Important QTL cluster}

Some QTL clusters for P-related traits have been mapped in the same genomic regions of wheat seedlings. For instance,
Su et al. (2006) found three QTL clusters for tiller number per plant, shoot dry weight, shoot $\mathrm{P}$ uptake, and shoot P-utilization efficiency on chromosomes 4B, 5A, and 5D, respectively. Guo et al. (2012) mapped 26 QTL clusters for six morphological and 18 nutrient $(\mathrm{N}, \mathrm{P}, \mathrm{K})$ efficiency traits on 13 chromosomes. Zhang and Wang (2015) identified 18 QTL clusters for 12 seedling traits that were mapped on 12 chromosomes under P-normal and P-deficient conditions. However, many QTL are only expressed in a given or a few environments. In this study, we found seven QTL clusters. Of these, $\mathrm{C} 4$ and $\mathrm{C} 5$ on chromosome $5 \mathrm{~B}$ were only detected in the NP1 and LP2 treatments, respectively; the other five QTL clusters (C1, C2, C3, C6, and C7) included 12 RHFQTL, which were important QTL clusters (Table 4, Fig. S1). They were discussed as follows.

Cluster C1 on chromosome 4D involved five QTL with contributions ranging from $9.74 \%$ to $11.84 \%$. Of these, three 
QTL ( $Q S d w-4 D, Q S p u t e-4 D$, and QTpute-4D) were RHFQTL. Expect for $Q R s d w-4 D$, four QTL were negative with increasing QTL effects that came from LM21, showing a favorable relationship between biomass traits (SDW and TDW) and PutE traits (SPutE and TPutE). This outcome illuminated that the biomass traits and PutE can be improved simultaneously at the seedling stage in wheat. The markers in the region ( $D-1124727$ and $D-1108531)$ may be valuable for further studies of the intrinsic regulatory mechanisms that controlling both PutE and biomass traits.

Cluster C2 on chromosome 5A comprised five QTL with contributions ranging from $11.08 \%(Q T d w-5 A)$ to $15.62 \%$ (QTpute-5A.1), indicating that all five QTL were major QTL. Among them, two QTL (QTdw-5A and QSpute-5A) were RHF-QTL. The LM6 alleles increased all five QTL effects, showing a favorable relationship between biomass traits (SDW and TDW) and PutE traits (SPutE, RPutE, and TPutE). In addition, the seven markers $D-1282770$, S-985909, D-1117080, D-100041125, D-1011689, S2292335 , and $S-1127707$ should be probably useful for lucubrating the interaction mechanism between PutE and biomass traits at the genomic level.

Three important clusters, C3, C6, and C7, were detected on chromosome 5B. C3 included five QTL with contributions ranging from $6.76(Q T d w-5 B .1)$ to $12.60 \%(Q S d w$ 5B.1). Four QTL ( $Q S d w-5 B .1, Q T d w-5 B .1, Q S p u t e-5 B .1$ and QTpute-5B.1) were RHF-QTL. LM21 alleles increased all five QTL, showing a favorable relationship between biomass traits (SDW and TDW) and PutE traits (RPutE, SPutE, and TPutE). Cluster C6 also involved five QTL with $R^{2}$ values different from 6.57 (QTpute.4) to $15.63 \%$ ( $Q S d w-5 B .4$ ), and two QTL were RHF-QTL ( $Q S d w-5 B .4$ and $Q T d w-5 B .4$ ). The LM6 alleles also increased all five QTL effects, showing a favorable relationship between the three biomass traits (RDW, SDW, and TDW) and two PutE traits (SPutE and TPutE). The above outcomes indicated that five markers in C3 (S-2283173, D-100082275, D-3031624, D-3023523, and $D$-3020625) and three markers in C6 (D-100091085, $D$-100010859, and D-3033994) provided reference information for in-depth study of key locus controlling PutE and biomass traits. Cluster C7 comprised three QTL for PupE traits (RPC, SPC, and TPC) with contributions ranging from 8.10\% (QRpc-5B.2) to $11.26 \%$ (QSpc-5B.4), and QTpc-5B was RHF-QTL. The additive effect of all three QTL came from LM6, demonstrating the positive relationship between the three PupE traits.

The seedling stage is an important period of wheat growth and development. In this study, detected 17 RHFQTL and five important QTL clusters controlling PE of wheat seedlings. Of these, 13 RHF-QTL were the main QTL with contributions over $10.00 \%$, which probably contribute to improve PE by biotechnological means in the future. Some RHF-QTL detected at seedling stage also could be constitutively expressed loci throughout plant growth and development. For example, we found five QTL clusters simultaneously for seedling and maturity traits, and a cluster on 4B (C5) including four RHF-QTLs with high contributions of 13.96-41.06\% in previous study (Yuan et al. 2017). Similarly, Kong et al. (2013) found 13 QTL clusters simultaneously for potassium $(\mathrm{K})$ efficiency traits at seedling and maturity stage. On the other hand, we also have to recognize that detecting the QTLs for PE at maturity stage are more sense for efficient phosphorus breeding. Therefore, it is necessary to further testing and finding out major QTL regulating PE of mature period in wheat, and the key QTL for controlling PE needs fine mapping and functional verification, in order to provide effective marker-assisted selection basis.

Acknowledgements This work was supported by the National Key R\&D Plan of China (2017YFD0100601) and the Modern Agricultural Industry Technology System of Shandong Province, China.

Author's Contributions FK and YA designed the experiments. YY, MZ, $\mathrm{HZ}$, YG, and YZ performed the experiments. YY analyzed the data. YY and YA wrote the paper.

\section{Compliance with ethical standards}

Conflict of interest No potential conflict of interest was reported by the authors.

Open Access This article is licensed under a Creative Commons Attribution 4.0 International License, which permits use, sharing, adaptation, distribution and reproduction in any medium or format, as long as you give appropriate credit to the original author(s) and the source, provide a link to the Creative Commons licence, and indicate if changes were made. The images or other third party material in this article are included in the article's Creative Commons licence, unless indicated otherwise in a credit line to the material. If material is not included in the article's Creative Commons licence and your intended use is not permitted by statutory regulation or exceeds the permitted use, you will need to obtain permission directly from the copyright holder. To view a copy of this licence, visit http://creativecommons.org/licenses/by/4.0/.

\section{References}

Baker A, Ceasar SA, Palmer AJ, Paterson JB, Qi W, Muench SP, Baldwin SA (2015) Replace, reuse, recycle: improving the sustainable use of phosphorus by plants. J Exp Bot 66:3523-3540. https://doi. org/10.1093/jxb/erv210

Bayuelo-Jiménez JS, Gallardo-Valdéz M, Pérez-Decelis VA, Magdaleno-Armas L, Ochoa I, Lynch JP (2011) Genotypic variation for root traits of maize (Zea mays L.) from the Purhepecha Plateau under contrasting phosphorus availability. Field Crops Res 121:350-362. https://doi.org/10.1016/j.fcr.2011.01.001

Bernardino KC, Pastina MM, Menezes CB, de Sousa SM, Maciel LS, Carvalho G Jr, Guimarães CT, Barros BA, E Silva LDC, Carneiro PC (2019) The genetic architecture of phosphorus efficiency in sorghum involves pleiotropic QTL for root morphology and grain 
yield under low phosphorus availability in the soil. BMC Plant Biol 19:87. https://doi.org/10.1186/s12870-019-1689-y

Churchill GA, Doerge RW (1994) Empirical threshold values for quantitative trait mapping. Genetics 138:963-971

Cui F, Zhao C, Ding A, Li J, Wang L, Li X, Bao Y, Li J, Wang H (2014) Construction of an integrative linkage map and QTL mapping of grain yield-related traits using three related wheat RIL populations. Theor Appl Genet 127:659-675. https://doi.org/10.1007/ s00122-013-2249-8

Dawson CJ, Hilton J (2011) Fertiliser availability in a resource-limited world: production and recycling of nitrogen and phosphorus. Food Policy 36:S14-S22. https://doi.org/10.1016/j.foodpol.2010.11.012

Dhillon J, Torres G, Driver E, Figueiredo B, Raun WR (2017) World phosphorus use efficiency in cereal crops. Agron J 109:16701677. https://doi.org/10.2134/agronj2016.08.0483

Gao M (2014) A high-density genetic map and QTLs for yield related traits in wheat. Ph.D, Dissertation, Shandong Agricultural University (in Chinese with English abstract)

Gong X, Liang X, Guo Y, Wu C, Zhao Y, Li X, Li S, Kong F (2015) Quantitative trait locus mapping for potassium use efficiency traits at the seedling stage in wheat under different nitrogen and phosphorus treatments. Crop Sci 55:2690-2700. https://doi. org/10.2135/cropsci2014.10.0711

Gunderson KL, Steemers FJ, Lee G, Mendoza LG, Chee MS (2005) A genome-wide scalable SNP genotyping assay using microarray technology. Nat Genet 37:549-554. https://doi.org/10.1016/j. tiv.2009.07.028

Guo Y, Kong F, Xu Y, Zhao Y, Liang X, Wang Y, An D, Li S (2012) QTL mapping for seedling traits in wheat grown under varying concentrations of N, P and K nutrients. Theor Appl Genet 124:851-865. https://doi.org/10.1007/s00122-011-1749-7

Hoagland DR, Arnon DI (1950) The water-culture method for growing plants without soil. Circ California Agricul Exp Stat 347:96

Kong F, Guo Y, Liang X, Wu C, Wang Y, Zhao Y, Li S (2013) Potassium (K) effects and QTL mapping for K efficiency traits at seedling and adult stages in wheat. Plant Soil 373:877-892. https:// doi.org/10.1007/s11104-013-1844-4

Liao M, Hocking PJ, Dong B, Delhaize E, Richardson AE, Ryan PR (2008) Variation in early phosphorus-uptake efficiency among wheat genotypes grown on two contrasting Australian soils. Aust J Agric Res 59:157-166. https://doi.org/10.1071/AR06311

López-Bucio J, Cruz-Ramırez A, Herrera-Estrella L (2003) The role of nutrient availability in regulating root architecture. Curr Opin Plant Biol 6:280-287. https://doi.org/10.1016/S1369 $-5266(03) 00035-9$

Ma W, Ma L, Li J, Wang F, Sisák I, Zhang F (2011) Phosphorus flows and use efficiencies in production and consumption of wheat, rice, and maize in China. Chemosphere 84:814-821. https://doi. org/10.1016/j.chemosphere.2011.04.055

Malhi SS, Vera CL, Brandt SA (2015) Seed yield potential of five wheat species/cultivars without and with phosphorus fertilizer application on a P-deficient soil in northeastern Saskatchewan. Agricul Sci 6:224. https://doi.org/10.4236/as.2015.62022

Meng X, Liu N, Zhang L, Yang J, Zhang M (2014) Genotypic differences in phosphorus uptake and utilization of watermelon under low phosphorus stress. J Plant Nutr 37:312-326. https://doi. org/10.1080/01904167.2013.852225

Nisar A, Khan SU, Shah AH (2016) Screening and evaluation of wheat germplasm for phosphorus use efficiency. Iran J Sci Technol Trans A Sci 40:201-207. https://doi.org/10.1007/s40995-016-0085-9

Péret B, Clément M, Nussaume L, Desnos T (2011) Root developmental adaptation to phosphate starvation: better safe than sorry. Trends Plant Sci 16:442-450. https://doi.org/10.1016/j.tplan ts.2011.05.006

Qiu H, Mei X, Liu C, Wang J, Wang G, Wang X, Liu Z, Cai Y (2013) Fine mapping of quantitative trait loci for acid phosphatase activity in maize leaf under low phosphorus stress. Mol Breed 32:629639. https://doi.org/10.1007/s11032-013-9895-z

Ryan PR, Liao M, Delhaize E, Rebetzke GJ, Weligama C, Spielmeyer W, James RA (2015) Early vigour improves phosphate uptake in wheat. J Exp Bot 66:7089-7100. https://doi.org/10.1093/jxb/ erv403

Schachtman DP, Reid RJ, Ayling SM (1998) Phosphorus uptake by plants: from soil to cell. Plant Physiol 116:447-453

Siddiqi MY, Glass AD (1981) Utilization index: a modified approach to the estimation and comparison of nutrient utilization efficiency in plants. J Plant Nutr 4:289-302

Su J, Xiao Y, Li M, Liu Q, Li B, Tong Y, Jia J, Li Z (2006) Mapping QTLs for phosphorus-deficiency tolerance at wheat seedling stage. Plant Soil 281:25-36. https://doi.org/10.1007/s1110 4-005-3771-5

Su J, Zheng Q, Li H, Li B, Jing R, Tong Y, Li Z (2009) Detection of QTLs for phosphorus use efficiency in relation to agronomic performance of wheat grown under phosphorus sufficient and limited conditions. Plant Sci 176:824-836. https://doi.org/10.1016/j.plant sci.2009.03.006

Syvänen A-C (2005) Toward genome-wide SNP genotyping. Nat Genet 37:S5-S10. https://doi.org/10.1038/ng1558

Vandamme E, Rose T, Saito K, Jeong K, Wissuwa M (2016) Integration of $\mathrm{P}$ acquisition efficiency, $\mathrm{P}$ utilization efficiency and low grain $\mathrm{P}$ concentrations into $\mathrm{P}$-efficient rice genotypes for specific target environments. Nutr Cycl Agroecosyst 104:413-427. https ://doi.org/10.1007/s10705-015-9716-3

Wang W, Ding G, White PJ, Wang X, Jin K, Xu F, Shi L (2018) Mapping and cloning of quantitative trait loci for phosphorus efficiency in crops: opportunities and challenges. Plant Soil 5:1-22. https://doi.org/10.1007/s11104-018-3706-6

Xin X, Qin S, Zhang J, Zhu A, Yang W, Zhang X (2017) Yield, phosphorus use efficiency and balance response to substituting longterm chemical fertilizer use with organic manure in a wheat-maize system. Field Crops Res 208:27-33. https://doi.org/10.1016/j. fcr.2017.03.011

Yuan Y, Gao M, Zhang M, Zheng H, Zhou X, Guo Y, Zhao Y, Kong F, Li S (2017) QTL mapping for phosphorus efficiency and morphological traits at seedling and maturity stages in wheat. Front Plant Sci 8:614. https://doi.org/10.3389/fpls.2017.00614

Zhang H, Wang H-g (2015) QTL mapping for traits related to P-deficient tolerance using three related RIL populations in wheat. Euphytica 203:505-520. https://doi.org/10.1007/s1068 1-014-1248-4 